Article

\title{
Starlikness Associated with Cosine Hyperbolic Function
}

\author{
Abdullah Alotaibi ${ }^{1}\left(\mathbb{D}\right.$, Muhammad Arif ${ }^{2, *}$, , Mohammed A. Alghamdi ${ }^{1}$ and Shehzad Hussain ${ }^{2}$ \\ 1 Operator Theory and Applications Research Group, Department of Mathematics, Faculty of Science, \\ King Abdulaziz University, Jeddah 21589, Saudi Arabia; mathker11@hotmail.com (A.A.); \\ Prof-malghamdi@hotmail.com (M.A.A.) \\ 2 Department of Mathematics, Abdul Wali Khan University Mardan, Mardan 23200, Pakistan; \\ shehzad873822@gmail.com \\ * Correspondence: marifmaths@awkum.edu.pk
}

Received: 3 June 2020; Accepted: 6 July 2020; Published: 8 July 2020

check for updates

\begin{abstract}
The main contribution of this article is to define a family of starlike functions associated with a cosine hyperbolic function. We investigate convolution conditions, integral preserving properties, and coefficient sufficiency criteria for this family. We also study the differential subordinations problems which relate the Janowski and cosine hyperbolic functions. Furthermore, we use these results to obtain sufficient conditions for starlike functions connected with cosine hyperbolic function.
\end{abstract}

Keywords: Janowski functions; subordination; cosine hyperbolic function

\section{Introduction and Definitions}

The aims of this particular section is to include some basic notions about the Geometric Function Theory that will help to understand our key findings in a clear way. In this regards, first we start to define the most basic family $\mathcal{A}$ which consists of holomorphic (or analytic) functions in $\mathcal{D}=$ $\{z \in \mathbb{C}:|z|<1\}$ by:

$$
\mathcal{A}=\left\{q: q \text { is holomorphic in } \mathcal{D} \text { with } q(z)=z+\sum_{k=2}^{\infty} a_{k} z^{k}\right\} .
$$

Also the set $\mathcal{S} \subset \mathcal{A}$ describes the family of all univalent functions which is define here by the following set builder form:

$$
\mathcal{S}=\{q \in \mathcal{A}: q \text { is univalent in } \mathcal{D}\}
$$

Next we consider defining the idea of subordinations between holomorphic functions $q_{1}$ and $q_{2}$, indicated by $q_{1} \prec q_{2}$, as; the functions $q_{1}, q_{2} \in \mathcal{A}$ are connected by the relation of subordination, if there exists a holomorphic function $v$ with the restrictions $v(0)=0$ and $|v(z)|<|z|$ such that $q_{1}(z)=q_{2}(v(z))$. Moreover, if the function $q_{2} \in \mathcal{S}$ in $\mathcal{D}$, then we obtain:

$$
q_{1} \prec q_{2} \Leftrightarrow q_{1}(0)=q_{2}(0) \& q_{1}(\mathcal{D}) \subset q_{2}(\mathcal{D})
$$

Image domains are of primary significance in the analysis of analytical functions. Analytic functions are classified into various families based on geometry of image domains. In 1992, Ma and Minda [1] considered a holomorphic function $\Delta$ normalized by the conditions $\Delta(0)=1$ and $\Delta^{\prime}(0)>0$ with $\operatorname{Re} \Delta>0$ in $\mathcal{D}$. The function $\Delta$ transforms the $\mathcal{D}$ disc into a region that is star-shaped about 1 and is symmetric on the real axis. In particular, if we take $\Delta(z)=\frac{1+L z}{1+M z}$ with $-1 \leq M<L \leq 1$, then it maps $\mathcal{D}$ to a disc which lies in the right-half plan with center on the real axis while $\frac{1-L}{1-M}$ and 
$\frac{1+L}{1+M}$ are its different end points of the diameter. This familiar function is recognized as Janowski function [2]. Some interesting problems such as convolution properties, coefficients inequalities, sufficient conditions, subordinates results, and integral preserving were discussed recently in [3-7] for some of the generalized families associated with circular domain. The image of the function $\Delta(z)=\sqrt{1+z}$ shows that the image domain is bounded by right-half plan of the Bernoullis lemniscate given by $\left|v^{2}-1\right|<1$, see [8]. The function $\Delta(z)=1+\frac{4}{3} z+\frac{2}{3} z^{2}$ maps $\mathcal{D}$ into the image set bounded by the cardioid

$$
\left(y^{2}+x^{2}-2 x+\frac{5}{9}\right)^{2}-16\left(y^{2}+x^{2}-\frac{2}{3} x+\frac{1}{9}\right)=0,
$$

which was examined in [9] and further studied in [10]. The function $\Delta(z)=1+\sin z$ was established by Cho and his coauthors in [11] while $\Delta(z)=e^{z}$ is recently studied in [12,13]. Furthermore, many subfamilies of starlike functions have also been introduced recently in [14-18] by choosing some particular functions such as functions associated with Bell numbers, functions related with shell-like curve connected with Fibonacci numbers, functions connected with conic domains and rational functions instead of the function $\Delta$.

Differential subordinations are natural generalizations in complex plane of differential inequalities on real line. Information obtained from derivative plays important role in studying properties of real valued functions. In complex plane, there are various differential implications, in which a function is characterized by using differential conditions. Noshiro-Warschawski theorem is an example of such differential implication which gives the univalency criterion for analytic functions. In numerous cases, properties of function are determined from the range of the combination of the derivatives of the function. For more details about differential subordinations, see [19].

Let $h$ be a holomorphic function defined on $\mathcal{D}$ with $h(0)=1$. Recently, Ali et al. have obtained sufficient conditions on $\lambda$ such that

$$
1+\lambda z h^{\prime}(z) / h^{n}(z) \prec \sqrt{1+z} \Rightarrow h(z) \prec \sqrt{1+z} \text {, for } n=0,1,2 \text {. }
$$

Similar type implications have been investigated in some of the recent papers by different researchers, for example see the articles contributed by Haq et al. [20], Kumar et al. [21,22], Paprocki and Sokół [23], Raza et al. [24], Sharma et al. [25] and Tuneski [26].

Now we establish the family $\mathcal{S}_{\text {cosh }}^{*}$ of starlike functions connected with cosine hyperbolic function that are defined by:

$$
\mathcal{S}_{\text {cosh }}^{*}=\left\{q \in \mathcal{A}: \frac{z q^{\prime}(z)}{q(z)} \prec \cosh (z),(z \in \mathcal{D})\right\} .
$$

Geometrically, the function $\frac{z q^{\prime}(z)}{q(z)}$ maps $\mathcal{D}$ onto an open disk symmetric with respect to the real axis with centre $\frac{\cosh (1)+\cos (1)}{2}$ and radius $\frac{\cosh (1)-\cos (1)}{2}$. It is interesting to see that the cosine and cosine hyperbolic functions have the same image domain in $\mathcal{D}$. For detail see [14].

Also, since $\cosh (z)$ maps the region $\mathcal{D}$ onto the image which is bounded by

$$
\left|\ln \left(v+\sqrt{v^{2}-1}\right)\right|<1 .
$$

Thus, the class $\mathcal{S}_{\text {cosh }}^{*}$ can also be defined in a different way as; a function $q \in \mathcal{A}$ belongs to the class $\mathcal{S}_{\text {cosh }}^{*}$ if and only if the following inequality will be true

$$
\left|\ln \left(\frac{z q^{\prime}(z)}{q(z)}+\sqrt{\left(\frac{z q^{\prime}(z)}{q(z)}\right)^{2}-1}\right)\right|<1 .
$$

We need to get the foregoing Lemma to establish our principal results. 
Lemma 1. [27] Let $v$ be a holomorphic function in $\mathcal{D}$ with $v(0)=0$. If

$$
\left|v\left(z_{0}\right)\right|=\max \left\{|v(z)| \text { for }|z| \leq\left|z_{0}\right|\right\}
$$

then a number $l(l \geq 1)$ occurs in such a way that $z_{0} v^{\prime}\left(z_{0}\right)=l v\left(z_{0}\right)$.

To avoid repetitions, we assume the following restrictions

$$
1 \leq M<L \leq 1, j \in \mathbb{N}=\{1,2, \ldots\}, k \in \mathbb{N}_{0}=\mathbb{N} \cup\{0\}
$$

otherwise we will state it where different.

\section{Sufficient Conditions Associated with Cosh}

Theorem 1. Let an analytic function $h($ with $h(0)=1)$ satisfying the relation of subordination

$$
1+\lambda\left(z h^{\prime}(z)\right)^{j} \prec \frac{1+L z}{1+M z},
$$

with the following limitation

$$
|\lambda| \geq \frac{(L-M)}{\sin ^{j}(1)-|M| \sinh ^{j}(1)},(\text { for } j \in \mathbb{N}) .
$$

Then

$$
h(z) \prec \cosh (z)
$$

Proof. Let us assume that

$$
p(z)=1+\lambda\left(z h^{\prime}(z)\right)^{j}
$$

Then the function $p$ is holomorphic in $\mathcal{D}$ with $p(0)=1$. Also consider

$$
v(z)=\cosh ^{-1}(h(z))
$$

where we selected the principle branches of the functions that are logarithmic and square root. Then $v$ is clearly a holomorphic function in $\mathcal{D}$ with $v(0)=0$. Also since

$$
\cosh ^{-1}(z)=\ln \left[z+\sqrt{z^{2}-1}\right]
$$

To complete the proof of this result, we just need to prove $|v(z)|<1$ in $\mathcal{D}$. By virtue of (7), we have

$$
p(z)=1+\lambda\left\{z v^{\prime}(z) \sinh (v(z))\right\}^{j} .
$$

Therefore

$$
\left|\frac{p(z)-1}{L-M p(z)}\right|=\left|\frac{\lambda\left\{z v^{\prime}(z) \sinh (v(z))\right\}^{j}}{(L-M)-\lambda M\left\{z v^{\prime}(z) \sinh (v(z))\right\}^{j}}\right| .
$$

Now, we suppose that a point $z_{0} \in \mathcal{D}$ occurs such that

$$
\max _{|z| \leq\left|z_{0}\right|}|v(z)|=\left|v\left(z_{0}\right)\right|=1
$$


Also, by Lemma 1 , a number $l \geq 1$ exists with $z_{0} v^{\prime}\left(z_{0}\right)=l v\left(z_{0}\right)$. In addition, we also suppose that $v\left(z_{0}\right)=e^{i \theta}$ for $\theta \in[-\pi, \pi]$. Then we have

$$
\begin{aligned}
\left|\frac{p\left(z_{0}\right)-1}{L-M p\left(z_{0}\right)}\right| & =\left|\frac{\lambda\left\{l v\left(z_{0}\right) \sinh \left(e^{i \theta}\right)\right\}^{j}}{(L-M)-\lambda M\left\{l v\left(z_{0}\right) \sinh \left(e^{i \theta}\right)\right\}^{j}}\right| \\
& \geq\left|\frac{|\lambda|\left\{l\left|\sinh \left(e^{i \theta}\right)\right|\right\}^{j}}{(L-M)+|\lambda||M|\left\{l\left|\sinh \left(e^{i \theta}\right)\right|\right\}^{j}}\right|
\end{aligned}
$$

If $|z|=r,-\pi \leq \theta \leq \pi$, then simple calculation illustrates that

$$
\begin{aligned}
& \left|\cosh \left(e^{i \theta}\right)\right|^{2}=\cosh ^{2}(\cos \theta) \cos ^{2}(\sin \theta)+\sinh ^{2}(\cos \theta) \sin ^{2}(\sin \theta)=\phi(\theta), \\
& \left|\sinh \left(e^{i \theta}\right)\right|^{2}=\sinh ^{2}(\cos \theta) \cos ^{2}(\sin \theta)+\cosh ^{2}(\cos \theta) \sin ^{2}(\sin \theta)=\mu(\theta) .
\end{aligned}
$$

A routine simplification ensures that $0, \pm \pi, \pm \frac{\pi}{2}$ are the roots of $\phi^{\prime}(\theta)=0$ and $\mu^{\prime}(\theta)=0$ in $[-\pi, \pi]$. Also, since

$$
\begin{aligned}
& \phi(\theta)=\phi(-\theta), \\
& \mu(\theta)=\mu(-\theta),
\end{aligned}
$$

it is enough to conclude that $\theta \in[0, \pi]$ and thus we achieve

$$
\begin{aligned}
\max \{\phi(\theta)\} & =\phi(0)=\phi(\pi)=\cosh ^{2}(1), \\
\min \{\phi(\theta)\} & =\phi\left(\frac{\pi}{2}\right)=\cos ^{2}(1), \\
\max \{\mu(\theta)\} & =\mu(0)=\mu(\pi)=\sinh ^{2}(1), \\
\min \{\mu(\pi)\} & =\mu\left(\frac{\pi}{2}\right)=\sin ^{2}(1) .
\end{aligned}
$$

Thus, we have

$$
\begin{aligned}
& \cos (1) \leq\left|\cosh \left(e^{i \theta}\right)\right| \leq \cosh (1) \\
& \sin (1) \leq\left|\sinh \left(e^{i \theta}\right)\right| \leq \sinh (1)
\end{aligned}
$$

Therefore, using (8)-(10), we attain

$$
\left|\frac{p\left(z_{0}\right)-1}{L-M p\left(z_{0}\right)}\right| \geq \frac{|\lambda| l^{j} \sin ^{j}(1)}{(L-M)+|\lambda||M| l^{j} \sinh ^{j}(1)} .
$$

Now let

$$
\varsigma(l)=\frac{|\lambda| l^{j} \sin ^{j}(1)}{(L-M)+|\lambda||M| l^{j} \sinh ^{j}(1)} .
$$

Then

$$
\varsigma^{\prime}(l)=\frac{|\lambda|(L-M) j^{j-1} \sin ^{j}(1)}{\left[(L-M)+|\lambda||M| l^{j} \sinh ^{j}(1)\right]^{2}}>0 .
$$

This confirms that the function $\varsigma$ is increasing and therefore $\varsigma(l) \geq \varsigma(1)$, so

$$
\left|\frac{p\left(z_{0}\right)-1}{L-M p\left(z_{0}\right)}\right| \geq \frac{|\lambda| \sin ^{j}(1)}{(L-M)+|\lambda||M| \sinh ^{j}(1)} .
$$


Now using (4), we achieve

$$
\left|\frac{p\left(z_{0}\right)-1}{L-M p\left(z_{0}\right)}\right| \geq 1
$$

and this contradicts the hypothesis

$$
1+\lambda\left(z h^{\prime}(z)\right)^{j} \prec \frac{1+L z}{1+M z}
$$

Hence the proof is completed.

If we put $h(z)=\frac{z q^{\prime}(z)}{q(z)}$ in (3), we achieve the below Corollary.

Corollary 1. Let $q \in \mathcal{A}$ and justifying

$$
1+\lambda\left\{z\left(\frac{z q^{\prime}(z)}{q(z)}\right)^{\prime}\right\}^{j} \prec \frac{1+L z}{1+M z} .
$$

with

$$
|\lambda| \geq \frac{(L-M)}{\sin ^{j}(1)-|M| \sinh ^{j}(1)} .
$$

Then $q \in \mathcal{S}_{\text {cosh }}^{*}$.

If we choose $L=1, M=0$ in (11), we get the following result.

Corollary 2. If $q \in \mathcal{A}$ and obeying the subordination

$$
1+\lambda\left\{z\left(\frac{z q^{\prime}(z)}{q(z)}\right)^{\prime}\right\}^{j} \prec 1+z .
$$

with

$$
|\lambda| \geq \frac{1}{\sin ^{j}(1)}
$$

Then $q \in \mathcal{S}_{\text {cosh }}^{*}$

Theorem 2. Let an analytic function $h(h(0)=1)$ satisfying the relation of subordination

$$
1+\lambda z \frac{h^{\prime}(z)}{h^{k}(z)} \prec \frac{1+L z}{1+M z}\left(\forall k \in \mathbb{N}_{0}\right),
$$

with the following restriction

$$
|\lambda| \geq \frac{(L-M) \cosh ^{k}(1)}{\sin (1)-|M| \sinh (1)} .
$$

Then

$$
h(z) \prec \cosh (z) .
$$

Proof. Let us suppose

$$
p(z)=1+\lambda \frac{z h^{\prime}(z)}{h^{k}(z)} .
$$

Then the function $p$ is holomorphic in $\mathcal{D}$ with $p(0)=1$. Inserting (7), we have

$$
p(z)=1+\lambda \frac{z v^{\prime}(z) \sinh (v(z))}{(\cosh v(z))^{k}} .
$$


and so

$$
\left|\frac{p(z)-1}{L-M p(z)}\right|=\left|\frac{\lambda z v^{\prime}(z) \sinh (v(z))}{(L-M) \cosh ^{k} v(z)-\lambda M\left\{z v^{\prime}(z) \sinh (v(z))\right\}}\right| .
$$

By virtue of Lemma 1 along with (9) and (10), we have

$$
\begin{aligned}
\left|\frac{p\left(z_{0}\right)-1}{L-M p\left(z_{0}\right)}\right| & =\left|\frac{\lambda l v\left(z_{0}\right) \sinh \left(v\left(z_{0}\right)\right)}{(L-M) \cosh ^{k}\left(v\left(z_{0}\right)\right)-\lambda M\left\{l v\left(z_{0}\right) \sinh \left(v\left(z_{0}\right)\right)\right\}}\right|, \\
& \geq \frac{l|\lambda|\left|\sinh \left(e^{i \theta}\right)\right|}{(L-\geq M)\left|\cosh ^{k}\left(e^{i \theta}\right)\right|+l|\lambda||M|\left|\sinh \left(e^{i \theta}\right)\right|}, \\
& \geq \frac{l|\lambda| \sin (1)}{(L-M) \cosh ^{k}(1)+l|\lambda||M| \sinh (1)} .
\end{aligned}
$$

Now let

$$
\varsigma_{1}(l)=\frac{l|\lambda| \sin (1)}{(L-M) \cosh ^{k}(1)+l|\lambda||M| \sinh (1)} .
$$

Then

$$
\varsigma_{1}^{\prime}(l)=\frac{|\lambda|(L-M) \cosh ^{k}(1) \sinh (1)}{\left\{(L-M) \cosh ^{k}(1)+l|\lambda||M| \sinh (1)\right\}^{2}}>0 .
$$

Applying (13), we have

$$
\left|\frac{p\left(z_{0}\right)-1}{L-M p\left(z_{0}\right)}\right| \geq 1
$$

A contradiction to the hypothesis occurs and hence the proof is completed.

If we take $h(z)=\frac{z q^{\prime}(z)}{q(z)}$ in (12), we obtain the below result.

Corollary 3. If $q \in \mathcal{A}$ and obeying the subordination

$$
1+\lambda z\left(\frac{q(z)}{z q^{\prime}(z)}\right)^{k}\left(\frac{z q^{\prime}(z)}{q(z)}\right)^{\prime} \prec \frac{1+L z}{1+M z}
$$

with

$$
|\lambda| \geq \frac{(L-M) \cosh ^{k}(1)}{\sin (1)-|M| \sinh (1)}
$$

then the function $q \in \mathcal{S}_{\text {cosh }}^{*}$.

If we choose $L=1, M=0$ in (14), we get the following result.

Corollary 4. If $q \in \mathcal{A}$ and obeying the subordination

$$
1+\lambda z\left(\frac{q(z)}{z q^{\prime}(z)}\right)^{k}\left(\frac{z q^{\prime}(z)}{q(z)}\right)^{\prime} \prec 1+z .
$$

with

$$
|\lambda| \geq \frac{\cosh ^{k}(1)}{\sin (1)}
$$

then $q \in \mathcal{S}_{\text {cosh }}^{*}$

Theorem 3. Assume that

$$
|\lambda| \geq \frac{(L-M) \cosh ^{k}(1)}{\sin ^{j}(1)-|M| \sinh ^{j}(1)}
$$


If $h$ is a holomorphc function defined on $\mathcal{D}$ with $h(0)=1$ and satisfying

$$
1+\lambda \frac{\left(z h^{\prime}(z)\right)^{j}}{h^{k}(z)} \prec \frac{1+L z}{1+M z}
$$

then

$$
h(z) \prec \cosh (z)
$$

Proof. Let us choose a function

$$
p(z)=1+\lambda \frac{\left(z h^{\prime}(z)\right)^{j}}{h^{k}(z)} .
$$

Then the function $p$ is holomorphic in $\mathcal{D}$ with $p(0)=1$. Applying some simple computation, we get

$$
p(z)=1+\lambda \frac{\left\{z v^{\prime}(z) \sinh (v(z))\right\}^{j}}{(\cosh v(z))^{k}}
$$

and so

$$
\begin{aligned}
\left|\frac{p(z)-1}{L-M p(z)}\right| & =\left|\frac{\lambda \frac{\left\{z v^{\prime}(z) \sinh (v(z))\right\}^{j}}{(\cosh (v(z)))^{k}}}{L-M\left\{1+\lambda \frac{\left\{z v^{\prime}(z) \sinh (v(z))\right\}^{j}}{(\cosh (v(z)))^{k}}\right\}}\right|, \\
& =\left|\frac{\lambda\left\{z v^{\prime}(z) \sinh (v(z))\right\}^{j}}{(L-M)(\cosh v(z))^{k}-\lambda M\left\{z v^{\prime}(z) \sinh (v(z))\right\}^{j}}\right| .
\end{aligned}
$$

By using Lemma 1, we have

$$
\begin{aligned}
\left|\frac{p\left(z_{0}\right)-1}{L-M p\left(z_{0}\right)}\right| & =\left|\frac{\lambda\left\{l v\left(z_{0}\right) \sinh \left(v\left(z_{0}\right)\right)\right\}^{j}}{(L-M) \cosh ^{k}\left(v\left(z_{0}\right)\right)-\lambda M\left\{l v\left(z_{0}\right) \sinh \left(v\left(z_{0}\right)\right)\right\}^{j}}\right| \\
& \geq\left|\frac{l^{j}|\lambda|\left|\sinh ^{j}\left(e^{i \theta}\right)\right|^{j}}{(L-M)\left|\cosh ^{k}\left(e^{i \theta}\right)\right|+l^{j}|\lambda||M|\left|\sinh ^{j}\left(e^{i \theta}\right)\right|}\right|, \\
& \geq \frac{l^{j}|\lambda| \sin ^{j}(1)}{(L-M) \cosh ^{k}(1)+l^{j}|\lambda||M| \sinh ^{j}(1)} .
\end{aligned}
$$

Now, let

$$
\varsigma_{2}(l)=\frac{l^{j}|\lambda| \sin ^{j}(1)}{(L-M) \cosh ^{k}(1)+l^{j}|\lambda||M| \sinh ^{j}(1)} .
$$

Then,

$$
\varsigma_{2}^{\prime}(l)=\frac{j^{l^{j-1}}|\lambda|(L-M) \sin ^{j}(1) \cosh ^{k}(1)}{\left\{(L-M) \cosh ^{k}(1)+l^{j}|\lambda||M| \sinh ^{j}(1)\right\}^{2}}>0,
$$

which shows that $\zeta$ is an increasing function and it has its minimum value at $l=1$, so

$$
\left|\frac{p\left(z_{0}\right)-1}{L-M p\left(z_{0}\right)}\right| \geq \frac{|\lambda| \sin ^{j}(1)}{(L-M) \cosh ^{k}(1)+|\lambda||M| \sinh ^{j}(1)} .
$$

Now by using (15), we have

$$
\left|\frac{p\left(z_{0}\right)-1}{L-M p\left(z_{0}\right)}\right| \geq 1
$$

which yields a contradiction to our assumption. This completes the proof. 
If we put $h(z)=\frac{z q^{\prime}(z)}{q(z)}$ in (16), we obtain the following result.

Corollary 5. If $q \in \mathcal{A}$ and obeying the subordination

$$
1+\lambda\left(\frac{q(z)}{z q^{\prime}(z)}\right)^{k}\left\{z\left(\frac{z q^{\prime}(z)}{q(z)}\right)^{\prime}\right\}^{j} \prec \frac{1+L z}{1+M z} .
$$

with

$$
|\lambda| \geq \frac{(L-M) \cosh ^{k}(1)}{\sin ^{j}(1)-|M| \sinh ^{j}(1)}
$$

then $q \in \mathcal{S}_{\text {cosh }}^{*}$.

If we choose $L=1, M=0$ in (17), we get the following result.

Corollary 6. If $q \in \mathcal{A}$ and obeying the subordination

$$
1+\lambda\left(\frac{q(z)}{z q^{\prime}(z)}\right)^{k}\left\{z\left(\frac{z q^{\prime}(z)}{q(z)}\right)^{\prime}\right\}^{j} \prec 1+z .
$$

with

$$
|\lambda| \geq \frac{\cosh ^{k}(1)}{\sin ^{j}(1)}
$$

Then $q \in \mathcal{S}_{\text {cosh }}^{*}$.

\section{Bernardi Integral Operator and Its Relationships}

The role of operators in the field of functions theory is very crucial in exploring the nature of the geometry of analytic functions. Several differential and integral operators were introduced by using convolution of certain analytic functions. It is found that this formalism gives ease in more mathematical study and also allows explaining the geometrical properties of analytical and univalent functions. Alexander was the first, who started studying the operator back in 1916. Later Libera [28] and Bernardi [29] added several integral operators to study the classes of starlike, convex, and close-to-convex functions. Also, the mapping properties of these operators was discussed in [30].

The Bernardi [29] integral operator is defined by;

$$
\mathcal{J}(z)=\frac{\xi+1}{z^{\tau}} \int_{0}^{z} t^{\xi-1} q(t) d t, \text { for } \xi \geq 0 .
$$

In this part of the article, we analyze the mapping properties of functions belonging to the class $\mathcal{S}_{\text {cosh }}^{*}$ under the integral operator described in (18) above. Some similar findings of this type are also discussed here.

Theorem 4. Assume that

$$
|\lambda| \geq \frac{(L-M)(\cosh (1)+\xi)}{\sin (1)-|N| \sinh (1)-(1+|M|) \cosh (1)(\cosh (1)+\xi)} .
$$

If

$$
1+\lambda z\left(\frac{z q^{\prime}(z)}{q(z)}\right) \prec \frac{1+L z}{1+M z}
$$


then

$$
\frac{z \mathcal{J}^{\prime}(z)}{\mathcal{J}(z)} \prec \cosh (z)
$$

where the operator $\mathcal{J}$ is given by (18).

Proof. Let a function $v$ be defined by

$$
v(z)=\cosh ^{-1}\left(\frac{z \mathcal{J}^{\prime}(z)}{\mathcal{J}(z)}\right) .
$$

where we have chosed the principle branches of the square root and logarithmic functions. Since $\cosh ^{-1} z$ function is defined by

$$
\cosh ^{-1} z=\ln \left[z+\left(z^{2}-1\right)^{1 / 2}\right],
$$

therefore $v$ is an analytic function in $\mathcal{D}$ with $v(0)=0$. To prove our result, we need only to show that $|v(z)|<1$ in $\mathcal{D}$. From (21), we have

$$
\frac{z \mathcal{J}^{\prime}(z)}{\mathcal{J}(z)}=\cosh (v(z))
$$

Logarithmic differentiation of above relation yields

$$
1+\frac{z \mathcal{J}^{\prime \prime}(z)}{\mathcal{J}^{\prime}(z)}-\frac{z \mathcal{J}^{\prime}(z)}{\mathcal{J}(z)}=\frac{z v^{\prime}(z) \sinh (v(z))}{\cosh (v(z))} .
$$

Using (18), we have

$$
(\xi+1) q(z)=z \mathcal{J}^{\prime}(z)+\xi \mathcal{J}(z) .
$$

Differentiating logarithmically, we have

$$
\begin{aligned}
\frac{z q^{\prime}(z)}{q(z)} & =\frac{z \mathcal{J}^{\prime}(z)}{\mathcal{J}(z)}\left\{\frac{1+\frac{z \mathcal{J}^{\prime \prime}(z)}{\mathcal{J}(z)}-\frac{z \mathcal{J}^{\prime}(z)}{\mathcal{J}(z)}}{\frac{z \mathcal{J}^{\prime}(z)}{\mathcal{J}(z)}+\xi}+1\right\} \\
& =\frac{z v^{\prime}(z) \sinh (v(z))+(\cosh (v(z))+\xi) \cosh (v(z))}{\cosh (v(z))+\xi}
\end{aligned}
$$

Now, we define a function

$$
\begin{aligned}
p(z) & =1+\lambda z\left(\frac{z q^{\prime}(z)}{q(z)}\right) \\
& =1+\lambda z\left\{\frac{z v^{\prime}(z) \sinh (v(z))+\cosh (v(z))(\cosh (v(z))+\xi)}{\cosh (v(z))+\xi}\right\},
\end{aligned}
$$

where $p$ is analytic in $\mathcal{D}$ with $p(0)=1$. Also

$$
\left|\frac{p(z)-1}{L-M p(z)}\right|=\left|\frac{\lambda z v^{\prime}(z) \sinh (v(z))+(\cosh (v(z))+\xi) \cosh (v(z))}{(L-M)\left(\cosh (v(z))+\xi^{\xi}\right)-\lambda \mathrm{M} z\left\{z v^{\prime}(z) \sinh (v(z))+(\cosh (v(z))+\xi) \cosh (v(z))\right\}}\right| .
$$

Suppose that there exists a point $z_{0} \in \mathcal{D}$ such that

$$
\max _{|z| \leq\left|z_{0}\right|}|v(z)|=\left|v\left(z_{0}\right)\right|=1 .
$$


By using Lemma 5 , there exists a number $l \geq 1$ such that $z_{0} v^{\prime}\left(z_{0}\right)=l v\left(z_{0}\right)$. We also suppose that $v\left(z_{0}\right)=e^{i \theta}$. Then we have

$$
\begin{aligned}
\left|\frac{p\left(z_{0}\right)-1}{L-M p\left(z_{0}\right)}\right| & =\left|\frac{\lambda z_{0}\left\{z_{0} v^{\prime}\left(z_{0}\right) \sinh \left(v\left(z_{0}\right)\right)+\cosh \left(v\left(z_{0}\right)\right)\left(\cosh \left(v\left(z_{0}\right)\right)+\xi\right)\right\}}{(L-M)\left(\cosh \left(v\left(z_{0}\right)\right)+\xi\right)-\lambda M z_{0}\left\{z_{0} v^{\prime}\left(z_{0}\right) \sinh \left(v\left(z_{0}\right)\right)+\cosh \left(v\left(z_{0}\right)\right)\left(\cosh \left(v\left(z_{0}\right)\right)+\xi\right)\right\}}\right| \\
& =\left|\frac{\lambda z_{0}\left\{l v\left(z_{0}\right) \sinh \left(e^{i \theta}\right)+\cosh \left(e^{i \theta}\right)\left(\cosh \left(e^{i \theta}\right)+\xi\right)\right\}}{(L-M)\left(\cosh \left(e^{i \theta}\right)+\xi\right)-\lambda M z_{0}\left\{l v\left(z_{0}\right) \sinh \left(e^{i \theta}\right)+\cosh \left(e^{i \theta}\right)\left(\cosh \left(e^{i \theta}\right)+\xi\right)\right\}}\right| .
\end{aligned}
$$

Let $|z|=r,-\pi \leq \theta \leq \pi$. Then a simple computation shows that

$$
\begin{aligned}
\left|\cosh \left(e^{i \theta}\right)\right| & =|\cosh (\cos \theta) \cos (\sin \theta)+i \sinh (\cos \theta) \sin (\sin \theta)| \\
\left|\cosh \left(e^{i \theta}\right)\right|^{2} & =\cosh ^{2}(\cos \theta) \cos ^{2}(\sin \theta)+\sinh ^{2}(\cos \theta) \sin ^{2}(\sin \theta)=\phi(\theta) .
\end{aligned}
$$

A simple computation shows that the equation $\phi^{\prime}(\theta)=0$ has five roots in $[-\pi, \pi]$ namely $0, \pm \pi, \pm \frac{\pi}{2}$. Since $\phi(\theta)=\phi(-\theta)$, it is sufficient to consider $\theta \in[0, \pi]$ and this implies that

$$
\begin{aligned}
\max \{\phi(\theta)\} & =\phi(0)=\phi(\pi)=\cosh ^{2}(1), \\
\min \{\phi(\theta)\} & =\phi\left(\frac{\pi}{2}\right)=\cosh ^{2}(1) .
\end{aligned}
$$

Also, consider

$$
\begin{aligned}
\left|\sinh \left(e^{i \theta}\right)\right| & =\sinh (\cos \theta) \cos (\sin \theta)+i \cosh (\cos \theta) \sin (\sin \theta) \\
\left|\sinh \left(e^{i \theta}\right)\right|^{2} & =\sinh ^{2}(\cos \theta) \cos ^{2}(\sin \theta)+\cosh ^{2}(\cos \theta) \sin ^{2}(\sin \theta)=\mu(\theta)
\end{aligned}
$$

Similarly, after simple calculations the equation $\mu^{\prime}(\theta)=0$ has five roots in $[-\pi, \pi]$ namely $0, \pm \pi, \pm \frac{\pi}{2}$. Since $\mu(\theta)=\mu(-\theta)$, it is sufficient to consider those roots which lies in $[0, \pi]$ and we see that

$$
\begin{aligned}
& \max \{\mu(\theta)\}=\mu(0)=\mu(\pi)=\cosh ^{2}(1)-1, \\
& \min \{\mu(\pi)\}=\mu\left(\frac{\pi}{2}\right)=1-\cos ^{2}(1) .
\end{aligned}
$$

Thus, we conclude that

$$
\begin{aligned}
& \cos (1) \leq\left|\cosh \left(e^{i \theta}\right)\right| \leq \cosh (1) \\
& \sin (1) \leq\left|\sinh \left(e^{i \theta}\right)\right| \leq \sinh (1)
\end{aligned}
$$

Now

$$
\begin{aligned}
\left|\frac{p\left(z_{0}\right)-1}{L-M p\left(z_{0}\right)}\right| & \geq \frac{|\lambda|\left\{\left|e^{i \theta}\right| l\left|e^{i \theta}\right|\left|\sinh \left(e^{i \theta}\right)\right|-\left|\cosh \left(e^{i \theta}\right)\right|\left(\left|\cosh \left(e^{i \theta}\right)\right|+\xi\right)\right\}}{(L-M)\left(\left|\cosh \left(e^{i \theta}\right)\right|+\xi\right)+|\lambda||M|\left|e^{i \theta}\right|\left\{l\left|e^{i \theta}\right|\left|\sinh \left(e^{i \theta}\right)\right|+\left|\cosh \left(e^{i \theta}\right)\right|\left(\left|\cosh \left(e^{i \theta}\right)\right|+\xi\right)\right\}} \\
& \geq \frac{|\lambda|\{l \sin (1)-\cosh (1)(\cosh (1)+\xi)\}}{(L-M)(\cosh (1)+\xi)+|\lambda||M|\{l \sinh (1)+\cosh (1)(\cosh (1)+\xi)\}} .
\end{aligned}
$$

Now let

$$
\Phi(l)=\frac{|\lambda|\{l \sin (1)-\cosh (1)(\cosh (1)+\xi)\}}{(L-M)(\cosh (1)+\xi)+|\lambda||M|\{l \sinh (1)+\cosh (1)(\cosh (1)+\xi)\}} .
$$


Then

$$
\Phi^{\prime}(l)=\frac{|\lambda|[(L-M)(\sin (1) \cosh (1)+\xi \sin (1))+|\lambda||M| \cosh (1)\{\sin (1)(1+\xi+\cosh (1))\}]}{[(L-M)(\cosh (1)+\xi)+|\lambda||M|\{l \sinh (1)+(\cosh (1)+\xi) \cosh (1)\}]^{2}}>0 .
$$

This shows that $\Phi$ is an increasing function and has its minimum value at $l=1$, so

$$
\left|\frac{p\left(z_{0}\right)-1}{L-M p\left(z_{0}\right)}\right| \geq \frac{|\lambda|\{\sin (1)-\cosh (1)(\cosh (1)+\xi)\}}{(L-M)(\xi+\cosh (1))+|\lambda||M|\{\sinh (1)+\cosh (1)(\cosh (1)+\xi)\}} .
$$

Now by (19), we have

$$
\left|\frac{p\left(z_{0}\right)-1}{L-M p\left(z_{0}\right)}\right| \geq 1
$$

A contradiction to the hypothesis

$$
1+\lambda z\left(\frac{z q^{\prime}(z)}{q(z)}\right) \prec \frac{1+L z}{1+M z}
$$

Hence we have the required result.

Theorem 5. Assume that

$$
|\lambda| \geq \frac{(L-M)(\xi+1)}{\sin (1)-|M| \sinh (1)-(1+|M|)(1+\xi) \cosh (1)} .
$$

If

$$
1+\lambda q(z) \prec \frac{1+L z}{1+M z}
$$

then

$$
\frac{\mathcal{J}(z)}{z} \prec \cosh (z)
$$

where $\mathcal{J}$ is the Bernardi integral operator defined in (18).

Proof. Let a function $v$ be defined by

$$
v(z)=\cosh ^{-1}\left(\frac{\mathcal{J}(z)}{z}\right)
$$

where we have chosed the principle branches of the square root and logarithmic functions. Then $v$ is analytic in $\mathcal{D}$ with $v(0)=0$. We need only to show that $|v(z)|<1$ in $\mathcal{D}$. From (23), we have

$$
\frac{\mathcal{J}(z)}{z}=\cosh (v(z))
$$

Also we define a function

$$
p(z)=1+\lambda q(z)
$$

where $p$ is analytic in $\mathcal{D}$ with $p(0)=1$. Now by using (18), (24) and (25), we have

$$
\left|\frac{p(z)-1}{L-M p(z)}\right|=\left|\frac{\lambda z\left\{z v^{\prime}(z) \sinh (v(z))+(1+\xi) \cosh (v(z))\right\}}{(L-M)(1+\xi)-\lambda z M\left\{z v^{\prime}(z) \sinh (v(z))+(1+\xi) \cosh (v(z))\right\}}\right| .
$$

Suppose that there exists a point $z_{0} \in \mathcal{D}$ such that

$$
\max _{|z| \leq\left|z_{0}\right|}|v(z)|=\left|v\left(z_{0}\right)\right|=1
$$


By using Lemma 5 , there exists a number $l \geq 1$ such that $z_{0} v^{\prime}\left(z_{0}\right)=l v\left(z_{0}\right)$. We also suppose that $v\left(z_{0}\right)=e^{i \theta}$. Then we have

$$
\begin{aligned}
\left|\frac{p\left(z_{0}\right)-1}{L-M p\left(z_{0}\right)}\right| & =\left|\frac{\lambda z_{0}\left\{z_{0} v^{\prime}\left(z_{0}\right) \sinh \left(v\left(z_{0}\right)\right)+(1+\xi) \cosh \left(v\left(z_{0}\right)\right)\right\}}{(L-M)(1+\xi)-\lambda z_{0} M\left\{z_{0} v^{\prime}\left(z_{0}\right) \sinh \left(v\left(z_{0}\right)\right)+(1+\xi) \cosh \left(v\left(z_{0}\right)\right)\right\}}\right| \\
& \geq \frac{|\lambda|\left\{l\left|\sinh \left(e^{i \theta}\right)\right|-(1+\xi)\left|\cosh \left(e^{i \theta}\right)\right|\right\}}{(L-M)(1+\xi)+|\lambda||M|\left\{l\left|\sinh \left(e^{i \theta}\right)\right|-(1+\xi)\left|\cosh \left(e^{i \theta}\right)\right|\right\}} \\
& \geq \frac{|\lambda|\{l \sin (1)-(1+\xi) \cosh (1)\}}{(L-M)(1+\xi)+|\lambda||M|\{l \sinh (1)+(1+\xi) \cosh (1)\}}
\end{aligned}
$$

Now let

$$
\Theta(l)=\frac{|\lambda|\{l \sin (1)-(1+\xi) \cosh (1)\}}{(L-M)(1+\xi)+|\lambda||M|\{l \sinh (1)+(1+\xi) \cosh (1)\}} .
$$

Then

$$
\Theta^{\prime}(l)=\frac{|\lambda|\{((L-M)(1+\xi) \sin (1)+|\lambda||M|(\sin (1)(1+\xi))+\sinh (1))\}}{(L-M)(1+\xi)+|\lambda||M|\{l \sinh (1)+(1+\xi) \cosh (1)\}^{2}}>0,
$$

which shows that $\Theta$ is an increasing function and it has its minimum value at $l=1$, so

$$
\left|\frac{p\left(z_{0}\right)-1}{L-M p\left(z_{0}\right)}\right| \geq \frac{|\lambda|\{\sin (1)-(1+\xi) \cosh (1)\}}{(L-M)(1+\xi)+|\lambda||M|\{\sinh (1)+(1+\xi) \cosh (1)\}} .
$$

Now by (22), we have

$$
\left|\frac{p\left(z_{0}\right)-1}{L-M p\left(z_{0}\right)}\right| \geq 1
$$

A contraduction to the hypothesis

$$
1+\lambda q(z) \prec \frac{1+L z}{1+M z} .
$$

Hence we have the required result.

Theorem 6. Assume that

$$
|\lambda| \geq \frac{(L-M)(1+\xi)}{\sin (1)-|M| \sinh (1)-(1+|M|)(1+\xi) \cosh (1)} .
$$

If

$$
1+\lambda z q^{\prime}(z) \prec \frac{1+L z}{1+M z},
$$

then

$$
\mathcal{J}^{\prime}(z) \prec \cosh (z),
$$

where $\mathcal{J}$ is the Bernardi integral operator defined in (18).

Proof. Using the same steps as used in the last result, one can easily complete this proof.

\section{Convolution Conditions and Its Consequences}

The technique of convolution (or Hadamard product) is extremely important in the solution of various function theory problems and due to this facts this concept becomes the major part of this field. The main goal of this portion is to analyze the properties of convolution and its implications 
for the family $\mathcal{S}_{\text {cosh }}^{*}$ of starlike functions subordinated with cosine hyperbolic function. For $q_{1}, q_{2} \in \mathcal{A}$, the convolution, denoted by $\left(q_{1} * q_{2}\right)(z)$, is defined by

$$
\left(q_{1} * q_{2}\right)(z)=z+\sum_{k=2}^{\infty} a_{k} b_{k} z^{k},(z \in \mathcal{D}) .
$$

Also, the following facts will be true only if $q \in \mathcal{A}$;

$$
q(z) * \frac{z}{1-z}=q(z) \text { and } q(z) * \frac{z}{(1-z)^{2}}=z q^{\prime}(z)
$$

Now using these concepts we now start to state and prove our first result.

Theorem 7. Let $q \in \mathcal{A}$. Then $q \in \mathcal{S}_{\text {cosh }}^{*}$ if and only if

$$
\frac{1}{z}\left[q(z) * \frac{z-\delta z^{2}}{(1-z)^{2}}\right] \neq 0,(z \in \mathcal{D}),
$$

for all $\delta=\delta_{\theta}=\frac{\cosh e^{i \theta}}{\cosh e^{i \theta}-1}$ and also for $\delta=1$.

Proof. Since given that $q \in \mathcal{S}_{\text {cosh }}^{*}$ is holomorphic in $\mathcal{D}$, it follows that $q(z) \neq 0$ for all $z \in \mathcal{D}^{*}=\mathcal{D} \backslash\{0\}$. That is $\frac{q(z)}{z} \neq 0$ for $z \in \mathcal{D}$ which is equivalent to (29) for $\delta=1$. Thus, the proof is completed for $\delta=1$. Now from (2), a holomorphic function $v$ occurs with the property that $v(0)=0$ and $|v(z)|<1$ so that

$$
\frac{z q^{\prime}(z)}{q(z)}=\cosh (v(z))
$$

and it is equivalent to

$$
\frac{z q^{\prime}(z)}{q(z)} \neq \cosh \left(e^{i \theta}\right), \text { for } \theta \in[0,2 \pi]
$$

Using (28), we can easily obtain

$$
q(z) * \frac{1}{(1-z)^{2}}-\cosh \left(e^{i \theta}\right)\left(\frac{q(z)}{z} * \frac{1}{(1-z)}\right) \neq 0,
$$

and then by simple computation, we have

$$
\frac{1}{z}\left[q(z) * \frac{z-\delta z^{2}}{(1-z)^{2}}\right] \neq 0,(z \in \mathcal{D}),
$$

which is the needed relationship.

For the converse part let assume that (29) hold for $\delta=1$, it implies that $\frac{q(z)}{z} \neq 0$ for all $z \in \mathcal{D}$. Thus, the function $\mathfrak{h}(z)=\frac{z q^{\prime}(z)}{q(z)}$ is holomorphic in $\mathcal{D}$ with $\mathfrak{h}(0)=1$. Also, let us take $\mathcal{H}(z)=\cosh \left(e^{i \theta}\right)$ for $z \in \mathcal{D}$ and since we have proven that (29) and (30) are identical, thus forming the relationship (30), it is evident that $\mathcal{H}(\partial \mathcal{D}) \cap \mathfrak{h}(\mathcal{D})=\phi$. Hence, a connected part of $\mathbb{C} \backslash \mathcal{H}(\partial \mathcal{D})$ contains the simply connected domain $\mathfrak{h}(\mathcal{D})$. The univalence of the function $\mathfrak{h}$, together with the fact $\mathcal{H}(0)=\mathfrak{h}(0)=1$, illustrates that $\mathfrak{h} \prec \mathcal{H}$ and it implies that $q \in \mathcal{S}_{\text {cosh }}^{*}$.

Theorem 8. Let $q \in \mathcal{A}$. Then a neccesary and sufficient condition $q \in \mathcal{S}_{\cosh }^{*}$ is that

$$
1-\sum_{n=2}^{\infty} \frac{n-\cosh \left(e^{i \theta}\right)}{\cosh \left(e^{i \theta}\right)-1} a_{n} z^{n-1} \neq 0, \quad(z \in \mathcal{D}) .
$$


Proof. In the last theorem, we have proved that $q \in \mathcal{S}_{\text {cosh }}^{*}$ if and only if the relation (29) held. We can rewrite (29) as

$$
\begin{aligned}
0 & \neq \frac{1}{z}\left[q(z) * \frac{z-\delta z^{2}}{(1-z)^{2}}\right] \\
& =\frac{1}{z}\left[q(z) *\left(\frac{z}{(1-z)^{2}}-\delta \frac{z^{2}}{(1-z)^{2}}\right)\right] \\
& =\frac{q(z)}{z} *\left(1+\sum_{n=2}^{\infty} n z^{n-1}-L \sum_{n=2}^{\infty}(n-1) z^{n-1}\right) \\
& =1-\sum_{n=2}^{\infty}((\delta-1) n-\delta) a_{n} z^{n-1} \\
& =1-\sum_{n=2}^{\infty} \frac{n-\cosh \left(e^{i \theta}\right)}{\cosh \left(e^{i \theta}\right)-1} a_{n} z^{n-1}
\end{aligned}
$$

and this completes the proof.

Theorem 9. If the function $q \in \mathcal{A}$ satisfies the following inequality

$$
\sum_{n=2}^{\infty}\left|\frac{n-\cosh \left(e^{i \theta}\right)}{\cosh \left(e^{i \theta}\right)-1}\right|\left|a_{n}\right|<1,
$$

then $q \in \mathcal{S}_{\text {cosh }}^{*}$.

Proof. To establish this result, we need to prove the relationship (31). For this consider

$$
\begin{aligned}
\left|1-\sum_{n=2}^{\infty}((\delta-1) n-\delta) a_{n} z^{n-1}\right| & >1-\sum_{n=2}^{\infty}\left|((\delta-1) n-\delta) a_{n} z^{n-1}\right| \\
& =1-\sum_{n=2}^{\infty}|((\delta-1) n-\delta)|\left|a_{n}\right||z|^{n-1} \\
& >1-\sum_{n=2}^{\infty}|((\delta-1) n-\delta)|\left|a_{n}\right| \\
& =1-\sum_{n=2}^{\infty}\left|\frac{n-\cosh \left(e^{i \theta}\right)}{\cosh \left(e^{i \theta}\right)-1}\right|\left|a_{n}\right|>0,
\end{aligned}
$$

where we have used inequality (32). Thus, by virtue of Theorem 8 , the proof is completed.

\section{Conclusions}

In the present research article, we examined some interesting properties of starlike functions associated with the cosine hyperbolic function which is symmetric about the real axis. These results included convolutions properties, Bernardi integral preserving problems and coefficient sufficiency criteria. In addition to that we also calculated some conditions on $\lambda$ so that; if for each $j \in \mathbb{N}$, $k \in \mathbb{N}_{0}=\mathbb{N} \cup\{0\}$

$$
1+\lambda \frac{\left(z h^{\prime}(z)\right)^{j}}{h^{k}(z)} \prec \frac{1+L z}{1+M z} \Rightarrow h(z) \prec \cosh (z),(z \in \mathcal{D}) .
$$

Furthermore, these results are used to find sufficiency criterion for the function belongs to the newly defined family $\mathcal{S}_{\text {cosh }}^{*}$. Moreover, some other problems like coefficient bounds, Hankel determinant, partial sum inequalities, and many more can be discussed for this class as a future work. 
Author Contributions: Conceptualization, A.A. and M.A.; Formal analysis, M.A. and S.H.; Funding acquisition, M.A.A.; Investigation, M.A.; Methodology, S.H. and A.A.; Software, M.A.A.; Supervision, M.A., A.A. and S.H.; Visualization, M.A.A.; Writing—original draft, M.A. and S.H.; Writing—review \& editing, M.A., A.A. and S.H. All authors have read and agreed to the published version of the manuscript.

Funding: This project was funded by the Deanship of Scientific Research (DSR) at King Abdulaziz University, Jeddah, under grant no. (RG-84-130-38).

Acknowledgments: This project was funded by the Deanship of Scientific Research (DSR) at King Abdulaziz University, Jeddah, under grant no. (RG-84-130-38). The authors, therefore, acknowledge with thanks DSR for technical and financial support.

Conflicts of Interest: The authors declare no conflict of interest.

\section{References}

1. Ma, W.; Minda, D. A unified treatment of some special classes of univalent functions. In Proceeding of the Conference on Complex Analysis; Li, Z., Ren, F., Yang, L., Zhang, S., Eds.; International Press: Vienna, Austria, 1994; pp. 157-169.

2. Janowski, W. Extremal problems for a family of functions with positive real part and for some related families. Ann. Pol. Math. 1970, 23, 159-177. [CrossRef]

3. Ahmad, K.; Arif, M.; Liu, J-.L. Convolution properties for a family of analytic functions involving $q$-analogue of Ruscheweyh differential operator. Turk. J. Math. 2019, 43, 1712-1720. [CrossRef]

4. Arif, M.; Ahmad, K.; Liu, J. L.; Sokół, J. A new class of analytic functions associated with Sălăgean operator. J. Funct. Spaces 2019, 2019, 5157394. [CrossRef]

5. Shi, L.; Khan, Q.; Srivastava, G.; Liu, J.-L.; Arif, M. A study of multivalent $q$-starlike functions connected with circular domain. Mathematics 2019, 7, 670. [CrossRef]

6. Srivastava, H.M.; Khan, B.; Khan, N.; Ahmad, Q.Z. Coeffcient inequalities for $q$-starlike functions associated with the Janowski functions. Hokkaido Math. J. 2019, 48, 407-425. [CrossRef]

7. Srivastava, H.M.; Tahir, M.; Khan, B.; Ahmad, Q.Z.; Khan, N. Some general classes of $q$-starlike functions associated with the Janowski functions. Symmetry 2019, 11, 292. [CrossRef]

8. Sokół, J.; Stankiewicz, J. Radius of convexity of some subclasses of strongly starlike functions. Zeszyty Nauk. Politech. Rzeszowskiej Mat 1996, 19, 101-105.

9. Sharma, K.; Jain, N.K.; Ravichandran, V. Starlike functions associated with a cardioid. Afrika Matematika 2016, 27, 923-939. [CrossRef]

10. Shi, L.; Ali, I.; Arif, M.; Cho, N.E.; Hussain, S.; Khan, H. A study of third Hankel determinant problem for certain subfamilies of analytic functions involving cardioid domain. Mathematics 2019, 7, 418. [CrossRef]

11. Cho, N.E.; Kumar, V.; Kumar, S.S.; Ravichandran, V. Radius problems for starlike functions associated with the sine function. Bull. Iran. Math. Soc. 2019, 45, 213-232. [CrossRef]

12. Mendiratta, R.; Nagpal, S.; Ravichandran, V. On a subclass of strongly starlike functions associated with exponential function. Bull. Malays. Math. Sci. Soc. 2015, 38, 365-386. [CrossRef]

13. Shi, L.; Srivastava, H.M.; Arif, M.; Hussain, S.; Khan H. An investigation of the third Hankel determinant problem for certain subfamilies of univalent functions involving the exponential function. Symmetry 2019, 11, 598. [CrossRef]

14. Bano, K.; Raza, M. Starlike functions associated with cosine functions. Bull. Iran. Math. Soc. 2020, revised.

15. Cho, N.E.; Kumar, S.; Kumar, V.; Ravichandran, V.; Srivastava, H.M. Starlike functions related to the Bell numbers. Symmetry 2019, 11, 219. [CrossRef]

16. Dziok, J.; Raina, R.K.; Sokół, J. On a class of starlike functions related to a shell-like curve connected with Fibonacci numbers. Math. Comput. Model. 2013, 57, 1203-1211. [CrossRef]

17. Kanas, S.; Răducanu, D. Some classes of analytic functions related to conic domains. Math. Slovaca 2014, 64, 1183-1196. [CrossRef]

18. Kumar, S.; Ravichandran, V. A subclass of starlike functions associated with a rational function. Southeast Asian Bull. Math. 2016, 40, 199-212.

19. Miller, S.S.; Mocanu, P.T. Differential Subordinations Theory and Its Applications; Marcel Dekker Inc.: New York, NY, USA; Basel, Switzerland, 2000.

20. Haq, M.; Raza, M.; Arif, M.; Khan, Q.; Tang, H. Q-analogue of differential subordinations. Mathematics 2019, 7, 724. [CrossRef] 
21. Kumar, S.S.; Kumar, V.; Ravichandran, V.; Cho, N.E. Sufficient conditions for starlike functions associated with the lemniscate of Bernoulli. J. Inequal. Appl. 2013, 2013, 176. [CrossRef]

22. Kumar, S.; Ravichandran, V. Subordinations for functions with positive real part. Complex Anal. Oper. Theory 2018, 12, 1179-1191. [CrossRef]

23. Paprocki, E.; Sokół, J. The extremal problems in some subclass of strongly starlike functions. Zeszyty Nauk. Politech. Rzeszowskiej Mat 1996, 20, 89-94.

24. Raza, M.; Sokół, J.; Mushtaq, S. Differential subordinations for analytic functions. Iran. J. Sci. Technol. Trans. A Sci. 2019, 43, 883-890. [CrossRef]

25. Sharma, K.; Ravichandran, V. Applications of subordination theory to starlike functions. Bull. Iran. Math. Soc. 2016, 42, 61-777.

26. Tuneski, N. Some simple sufficient conditions for starlikeness and convexity. Appl. Math. Lett. 2009, 22, 693-697. [CrossRef]

27. Jack, I.S. Functions starlike and convex of order alpha. J. Lond. Math. Soc. 1971, 2, 469-474. [CrossRef]

28. Libera, R.J. Some classes of regular univalent functions. Proc. Am. Math. Soc. 1965, 16, 755-758. [CrossRef]

29. Bernardi, S.D. Convex and starlike univalent functions. Trans. Am. Math. Soc. 1969, 135, 429-446. [CrossRef]

30. Noor, K.I.; Arif, M. Mapping properties of an integral operator. Appl. Math. Lett. 2012, 25, 1826-1829. [CrossRef]

(C) 2020 by the authors. Licensee MDPI, Basel, Switzerland. This article is an open access article distributed under the terms and conditions of the Creative Commons Attribution (CC BY) license (http:/ / creativecommons.org/licenses/by/4.0/). 